\section{Eventos de vida produtores de estresse e queixas de insônia entre auxiliares de enfermagem de um hospital universitário no Rio de Janeiro: Estudo Pró-Saúde}

\section{Stressful life events and insomnia complaints among nursing assistants from a university hospital in Rio de Janeiro: The Pro-Saude Study}

Jaqueline R. Robaina'

Claudia S. Lopes'

Lúcia Rotenberg"

Eduardo Faerstein'

Frida M. Fischer"II

Claudia R. de C. Moreno"'I

Guilherme L. Werneck'

Dora Choriv

'Departamento de Epidemiologia do Instituto de Medicina Social da Universidade do Estado do Rio de Janeiro (UERJ).

"Laboratório de Educação em Ambiente e Saúde do Departamento de Biologia do Instituto Oswaldo Cruz da Fundação Oswaldo Cruz (FIOCRUZ).

"'Departamento de Saúde Ambiental da Faculdade de Saúde Pública da Universidade de São Paulo (USP).

IV Departamento de Epidemiologia e Métodos Quantitativos em Saúde de Escola Nacional de Saúde Pública Sérgio Arouca da Fundação Oswaldo Cruz (FIOCRUZ).

Financiamento: Este trabalho foi parcialmente financiado pela Fundação de Amparo à Pesquisa do Rio de Janeiro (FAPERJ) Nº processo: E-26/151.753/2003.

Conflito de interesses: inexistente.

Agradecimentos: À toda a equipe do Pró-Saúde, que participou das etapas do trabalho de campo e digitação dos dados, e aos funcionários técnico-administrativos da universidade que responderam aos questionários desta pesquisa.

Correspondência: Claudia S. Lopes. Departamento de Epidemiologia do Instituto de Medicina Social da Universidade do Estado do Rio de Janeiro (IMS-UERJ), Rua São Francisco Xavier, 524, 70 andar , Rio de Janeiro, RJ CEP 20559-900. E-mail: lopes@ims.uerj.br

\section{Resumo}

Objetivo: Avaliar a associação entre eventos de vida produtores de estresse (EVPE) e queixas de insônia (QI). Métodos: Foram analisados dados seccionais de 695 auxiliares de enfermagem de um hospital universitário, participantes do Estudo PróSaúde - coorte de funcionários de uma universidade no Rio de Janeiro. As informações foram obtidas através de um questionário multidimensional e autopreenchido, que avaliou a ocorrência de EVPE nos últimos 12 meses, variáveis socioeconômicas e demográficas e QI. As QI foram analisadas como desfecho politômico (frequente, ocasional, e ausente). Odds ratios brutos e ajustados foram calculados através de regressão logística multinomial. Resultados: A prevalência total de QI foi de 45,8\% (16,7\% frequentes e $29,1 \%$ ocasionais). Após ajuste por sexo, idade, estado civil, renda familiar per capita e regime de trabalho, os EVPE associados com QI frequentes foram: "rompimento de relação amorosa" (OR = 3,32; IC95\% 1,90 $5,78)$, "ter tido problemas graves de saúde" $(\mathrm{OR}=2,82$; IC95\% 1,73 - 4,58); "dificuldades financeiras graves" $(\mathrm{OR}=2,38$; IC95\% 1,46 $3,88)$, e "mudança forçada de moradia" (OR = 1,97; IC95\% 1,02 - 3,79). Com relação às QI ocasionais, houve associação apenas com rompimento de relação amorosa $(\mathrm{OR}=2,30$; IC95\% 1,42 - 3,74) e dificuldades financeiras graves $(\mathrm{OR}=1,87$; IC95\% $1,27-2,75)$. Conclusões: Dada a responsabilidade com vidas humanas que os auxiliares de enfermagem assumem durante seu horário de trabalho, nossos achados podem contribuir para ações mais efetivas, por parte dos serviços de saúde ao trabalhador, para lidar com o estresse nessa categoria.

Palavras-chave: Insônia. Sono. Queixas de Insônia. Eventos de Vida Produtores de Estresse. Saúde do Trabalhador. Auxiliares de Enfermagem. Epidemiologia. 


\section{Abstract}

Objective: To evaluate the association between stressful life events (SLE) and insomnia complaints (IC). Methods: Crosssectional data from 695 certified nursing assistants from a university hospital, participants of the Pro-Saude Study - a cohort of university employees in Rio de Janeiro, were analyzed. Information was obtained through a self-administered multidimensional questionnaire, which evaluated the occurrence of SLE in the previous 12 months, socioeconomic and demographic variables, and IC. Insomnia complaints were analyzed as a polythomic outcome (frequent, occasional, and absent). Crude and adjusted odds ratios were calculated through multinomial logistic regression. Results: Total prevalence of IC was $45.8 \%$ (frequent $16.7 \%$ and occasional $29.1 \%$ ). After adjusting for sex, age, marital status, per capita family income, and work hours, the SLE associated with frequent IC were: "breakup of a love relationship" (OR $=3.32$; 95\%CI 1.90-5.78), "having had severe health problems" (OR=2.82; 95\%CI 1.73-4.58), "severe financial problems" (OR=2.38; 95\%CI 1.46-3.88), and "forced change of residence" (OR=1.97; 95\%CI 1.02-3.79). Occasional IC was associated only with "breakup of a love relationship" (OR=2.30; 95\%CI 1.42-3.74) and "severe financial problems" (OR=1.87; 95\%CI 1.27-2.75). Conclusions: Given the responsibility over human lives taken on by nursing assistants during their work hours, our findings may contribute to more effective actions to be taken by health services toward workers in this category, helping them better deal with stress.

Keywords: Insomnia. Sleep. Insomnia Complaints. Stressful Life Events. Occupational Health. Nursing Assistants. Epidemiology.

\section{Introdução}

A insônia é o transtorno do sono mais frequente na população em geral, sendo reconhecida pela OMS como um problema de saúde pública ${ }^{1}$ devido ao impacto negativo à saúde física e mental, atividade social, capacidade para o trabalho e qualidade de vida dos indivíduos ${ }^{2-5}$. Apesar disso, este tema não tem recebido a devida atenção dos profissionais da área da saúde e os próprios insones, na maioria dos casos, não procuram ajuda profissional qualificada ${ }^{2,6,7}$.

Levantamentos bibliográficos mostram grande variação nas estimativas de prevalência da insônia, como observam Rocha e Costa $(2000)^{3}$ e Souza e Reimão (2004) ${ }^{8}$. Entre os fatores metodológicos aos quais os autores atribuem tal variabilidade, destacam-se o critério de definição da insônia (presença do sintoma, nível de severidade, frequência ou duração), a amplitude das respostas, a faixa etária e o processo de amostragem. Morin e cols. (2006) ${ }^{7}$ observam prevalências mais baixas quando do uso de critérios mais restritos para a definição de insônia.

A maior prevalência de insônia entre mulheres é o achado mais consistente na literatura sobre distúrbios do sono ${ }^{9-11}$. O aumento da prevalência com a idade também tem sido observado ${ }^{11}$.

A OMS preconiza a realização de pesquisas voltadas para a identificação da prevalência deste transtorno em países em desenvolvimento e sua associação com variáveis demográficas ${ }^{1}$. Tais estudos ainda são escassos no Brasil. Rocha e cols. (2002) ${ }^{12}$, em Bambuí (MG), encontraram uma prevalência de $35,4 \%$ de insônia na população acima de 18 anos, considerando o relato referente ao mês anterior à entrevista. Em São Paulo, cerca de $50 \%$ dos entrevistados relataram alguma queixa de insônia ao menos uma vez na semana ${ }^{13}$. Pesquisa realizada em Campo Grande (MS) com amostra representativa da população geral acima de 18 anos mostrou uma prevalência de insônia de 19,1\%, com base na definição adotada pela ASDA, Associação Americana de Transtornos do Sono ${ }^{14}$. 
A ocorrência de eventos de vida produtores de estresse está entre os fatores associados à insônia ${ }^{15-18}$. Segundo Monti $(2000)^{19}$, a maioria dos casos de insônia primária (aquela cuja etiologia não está relacionada a outro transtorno mental, condição médica ou dependência de substâncias) pode ser induzida por situações de estresse, como o afastamento de um familiar, perda do emprego, dificuldade econômica, intervenção cirúrgica, etc.

A identificação de queixas relacionadas ao sono entre profissionais de enfermagem é relevante por se tratar de categoria em cujo ambiente de trabalho acidentes de pequeno porte podem pôr em risco a vida das pessoas sob seus cuidados. A avaliação da relação entre eventos de vida produtores de estresse e queixas de insônia poderá, eventualmente, subsidiar atividades na área voltadas para lidar com o estresse, como parte das ações da saúde do trabalhador em hospitais.

O objetivo desse estudo é estimar a prevalência de queixas de insônia e avaliar sua associação a eventos de vida produtores de estresse entre auxiliares de enfermagem de um hospital universitário.

\section{Material e métodos}

\section{Desenho e população de estudo}

Este trabalho insere-se na Fase 1 (1999) do Estudo Pró-Saúde ${ }^{20}$, um estudo de coorte cujo objetivo geral é investigar o papel de determinantes biológicos e socioculturais nos padrões de saúde e de morbidade de funcionários técnico-administrativos efetivos de uma universidade pública no Estado do Rio de Janeiro. O presente trabalho utiliza um delineamento seccional para avaliar a associação entre eventos de vida produtores de estresse (EVPE) e queixas de insônia.

A população alvo deste estudo são todos os auxiliares de enfermagem em atividade lotados no hospital universitário. Excluindose os funcionários em licença não-médica, aposentados e cedidos a outras instituições, a população elegível para participar do estudo foi composta de 4.177 funcionários, dos quais $4.030(90,4 \%)$ responderam ao questionário. Após exclusão dos indivíduos que não responderam às perguntas sobre queixas de insônia (5,95\%), a população final de estudo foi de 695 auxiliares de enfermagem.

\section{Instrumentos}

Foram utilizados questionários autopreenchíveis, aplicados por entrevistadores treinados ${ }^{21}$. Neste estudo são avaliadas as questões relativas às queixas de insônia, dados socioeconômicos, demográficos $\mathrm{e}$ relativos ao trabalho e os eventos de vida produtores de estresse.

Queixas de insônia: Nesse estudo, trabalhamos com a variável "queixa de insônia" e não "insônia”, pois no questionário utilizado as questões relativas ao sono não contemplam os critérios dos principais sistemas de classificação para o diagnóstico da insônia, quais sejam: o Diagnostic and Statistical Manual of Mental Diseases (DSM-IV, 1994)22, a International Classification of Sleep Disorders (AASM, 2001) ${ }^{23} \mathrm{e}$ a Classificação Internacional de Doenças, versão 10, CID-10 (WHO, 1997) ${ }^{24}$.

A avaliação das queixas de insônia se baseia em critérios de frequência, que é o mais utilizado na literatura (Ohayon, $2002)^{25}$, a partir das seguintes perguntas "Nas ÚLTIMAS DUAS SEMANAS, com que frequência você teve dificuldade para pegar no sono?" e "Nas ÚLTIMAS DUAS SEMANAS, com que frequência você acordou durante o sono e teve dificuldade para dormir de novo?" Utilizou-se uma escala do tipo Likert para avaliar as respostas a essas perguntas (sempre, quase sempre, às vezes, raramente ou nunca).

A variável foi categorizada em:

- "queixa de insônia frequente", quando o respondente marcava "sempre" e/ou "quase sempre" em pelo menos uma das perguntas;

- "queixa de insônia ocasional", quando respondia "às vezes" às duas questões relativas à insônia, ou "às vezes" a uma das questões e "quase nunca" ou "nunca" a outra; e 
- "sem queixa de insônia", quando respondia "raramente" e/ou "nunca" às duas perguntas.

Variáveis socioeconômicas, demográficas e relativas ao regime de trabalho: Foram avaliadas as seguintes variáveis socioeconômicas e demográficas: sexo, idade, situação conjugal, grau de instrução e renda familiar per capita em salários mínimos (SM). A pergunta sobre renda foi originalmente estruturada em faixas de valores expressas em Reais, sendo a média dos valores de cada categoria dividida pelo salário mínimo da época, $\mathrm{R} \$ 136,00$.

A questão relativa ao regime de trabalho foi avaliada com base na questão "Atualmente, você trabalha em algum plantão noturno ou de 24 horas, no hospital universitário, campus da universidade, ou fora da universidade?" Com opções de resposta sim e não, dando origem a uma variável com duas categorias: realiza plantão noturno / não realiza plantão noturno.

Eventos de Vida Produtores de Estresse (EVPE): Foram utilizadas perguntas simples e breves, que compunham os chamados "eventos de vida produtores de estresse" (do inglês, stressful life events), de acordo com o procedimento descrito por Lopes e Faerstein $(2001)^{26}$ e Lopes e cols. (2003) ${ }^{27}$. O período de referência para a ocorrência do evento eram os 12 meses anteriores ao preenchimento do questionário. Os EVPE avaliados foram: problemas de saúde que impediram a realização de atividades habituais (trabalho, estudo ou lazer) por mais de um mês; internação hospitalar por uma noite, ou mais, em razão de doença ou acidente; falecimento de algum parente próximo (pai, mãe, cônjuge, companheiro(a), filho ou irmão); dificuldades financeiras mais severas do que as habituais; mudança forçada de moradia (por exemplo, devido a aumento do aluguel); rompimento de relação amorosa, incluindo divórcio ou separação; assalto ou roubo, isto é, ter tido dinheiro ou algum bem tomado, mediante uso ou ameaça de violência; ter sido vítima de alguma agressão física ${ }^{26,27}$.

\section{Aspectos éticos}

A pesquisa na qual o presente trabalho se insere foi submetida e aprovada nos Comitês de Ética da própria universidade onde se realizou o estudo, e do hospital universitário.

\section{Análise dos dados}

Na primeira etapa foi realizada uma descrição geral dos dados através do exame da distribuição de frequências para cada variável de estudo. Todas as análises foram conduzidas considerando o desfecho em estudo (queixas de insônia) como politômico (três categorias), sendo estas as categorias: frequente, ocasional e sem queixa de insônia.

Foram realizadas análises bivariadas para avaliar a associação entre as variáveis explicativas (EVPE) e a variável dependente (queixas de insônia). A associação da variável dependente com as variáveis explicativas foi expressa através dos odds ratios (OR) e seus respectivos intervalos de $95 \%$ de confiança. Para avaliar a significância estatística das associações foram utilizados os testes de qui-quadrado de Pearson.

A análise multivariada foi feita através de regressão logística multinomial, com cálculo dos odds ratios (OR) simples e ajustados. Foram incluídas nos modelos as variáveis que mostraram associação simples com desfecho com $\mathrm{p} \leq 0,25^{28}$. Caso este critério levasse à exclusão de variáveis relevantes, segundo o que consta na literatura sobre o tema, a inclusão destas foi considerada. Permaneceram no modelo as variáveis independentes que mantiveram associação com desfecho após ajuste ( $\mathrm{p} \leq 0,05)$, de acordo com teste de razão de verossimilhança (likelihood ratio test).

\section{Resultados}

A população do estudo foi constituída por 695 auxiliares de enfermagem, dentre os quais $140(20,1 \%)$ eram homens e $555(79,9 \%)$ eram mulheres. Um maior percentual de auxiliares de enfermagem 
apresentou idade entre 30 a 49 anos (80,2\%). Pouco mais da metade da população eram casados (55,7\%), 20,7\% solteiros e $19,8 \%$ separados/divorciados. Cerca de $44 \%$ da amostra encontrava-se no segundo estrato de renda familiar per capita (de 3 a 6 salários mínimos), metade da população apresentou nível educacional igual ou superior ao $2^{\circ}$ grau completo $(51,5 \%)$. Um percentual de $43,5 \%$ trabalhava em regime de plantão noturno.

A distribuição dos indivíduos da amostra pelas variáveis socioeconômicas e demográficas, e relativas ao horário de trabalho, segundo o padrão de queixas de insônia, está descrita na Tabela 1. Observamos uma maior prevalência de queixas de insônia, tanto frequente como ocasional, nas mulheres, entre aqueles com renda familiar per capita mais baixa (até três salários mínimos) e entre os separados/divorciados. Os funcionários que relataram trabalhar em regime de plantão apresentaram uma prevalência mais elevada de queixas de insônia ocasional, mas não frequente.

A prevalência de queixas de insônia segundo o relato de eventos de vida produtores de estresse está descrita na Tabela

Tabela 1 - Prevalência de queixas de insônia segundo características socioeconômicas e demográficas e relativas ao regime de trabalho, entre auxiliares de enfermagem de um hospital universitário no Rio de Janeiro. Estudo Pró-Saúde, 1999.

Table 1 - Prevalence of insomnia complaints according to socioeconomic and demographic characteristics and work hours among nursing assistants from a university hospital in Rio de Janeiro. The Pro-Saude Study, 1999.

\begin{tabular}{|c|c|c|c|c|}
\hline & \multirow{2}{*}{$\begin{array}{c}\text { Sem queixas } \\
\text { de insônia } \\
n(\%)\end{array}$} & \multicolumn{2}{|c|}{ Queixas de insônia } & \multirow[b]{2}{*}{ p-valor } \\
\hline & & $\begin{array}{c}\text { Ocasional } \\
\mathrm{n}(\%)\end{array}$ & $\begin{array}{c}\text { Frequente } \\
\mathrm{n}(\%)\end{array}$ & \\
\hline & $377(54,2)$ & $202(29,1)$ & $116(16,7)$ & \\
\hline \multicolumn{5}{|l|}{ Sexo } \\
\hline Masculino & $94(67,1)$ & $27(19,3)$ & $19(13,6)$ & 0,002 \\
\hline Feminino & $283(51,0)$ & $175(31,5)$ & $97(17,5)$ & \\
\hline \multicolumn{5}{|l|}{ Idade } \\
\hline 20-29 anos & $27(58,7)$ & $13(28,3)$ & $6(13,0)$ & 0,037 \\
\hline 30-39 anos & $181(59,7)$ & $85(28,0)$ & $37(12,2)$ & \\
\hline 40-49 anos & $129(50,8)$ & $73(28,7)$ & $52(20,5)$ & \\
\hline 50 anos ou mais & $40(43,5)$ & $31(33,7)$ & $21(22,8)$ & \\
\hline \multicolumn{5}{|l|}{ Renda familiar per capita } \\
\hline Até 3 Salários Mínimos & $107(46,7)$ & $76(33,2)$ & $46(20,1)$ & 0,044 \\
\hline Entre 3 e 6 SM & $173(49,9)$ & $78(27,0)$ & $38(13,1)$ & \\
\hline Mais de 6 SM & $76(56,3)$ & $37(27,4)$ & $22(16,3)$ & \\
\hline \multicolumn{5}{|l|}{ Grau de instrução } \\
\hline $1^{\circ} \mathrm{Grau}$ incompleto & $7(58,3)$ & $3(25,0)$ & $2(16,67)$ & 0,059 \\
\hline $1 \circ \mathrm{Grau}$ completo & $85(45,2)$ & $63(33,5)$ & $40(21,3)$ & \\
\hline $2^{\circ} \mathrm{Grau}$ completo & $199(56,2)$ & $95(26,8)$ & $60(16,9)$ & \\
\hline Universitário completo/Pós-graduação & $80(60,1)$ & $40(30,1)$ & $13(9,8)$ & \\
\hline \multicolumn{5}{|l|}{ Situação conjugal } \\
\hline Casado/vivendo em união & $211(57,6)$ & $96(26,2)$ & $59(16,1)$ & 0,048 \\
\hline Separado/Divorciado & $54(41,5)$ & $45(34,6)$ & $31(23,8)$ & \\
\hline Solteiro & $76(60,0)$ & $42(28,0)$ & $18(12,0)$ & \\
\hline Viúvo & $15(55,9)$ & $7(30,9)$ & $3(13,2)$ & \\
\hline \multicolumn{5}{|l|}{ Realiza plantão noturno } \\
\hline Sim & $178(59,9)$ & $69(23,2)$ & $50(16,8)$ & 0,019 \\
\hline Não & $197(51,0)$ & $127(32,9)$ & $62(16,1)$ & \\
\hline
\end{tabular}


2. As diferenças entre os grupos foram estatisticamente significativas $(\mathrm{p}<0,05)$ com relação à maioria dos eventos, exceto quanto a ter sido internado em hospital, ter sido vítima de assalto ou roubo, ou de agressão física.

Após ajuste por sexo, idade, estado civil, renda familiar per capita e regime de trabalho, observou-se que aqueles com história de rompimento de relação amorosa, problemas de saúde, dificuldade financeira grave e mudança forçada de moradia apresentavam prevalência significativamente mais elevada de queixa de insônia frequente quando comparados àqueles que não relataram exposição aos EVPE investigados. Ter sido vítima de agressão física e ter sido internado em hospital mostraram associações marginalmente significativas com queixas de insônia. Entretanto, quando se compara o grupo que refere queixa de insônia ocasional com o grupo que não apresenta queixa de insônia, apenas rompimento de relação amorosa e dificuldade financeira grave se mantiveram associadas com insônia de uma forma estatisticamente significante. Falecimento de parente próximo mostrou associação marginalmente significativa, com insônia ocasional (Tabela 2).

Tabela 2 - Prevalência de queixas de insônia e odds ratios (OR) brutos e ajustados e respectivos intervalos de 95\% de confiança (IC 95\%) da associação entre eventos de vida produtores de estresse (EVPE) e queixas de insônia entre auxiliares de enfermagem de um hospital universitário no Rio de Janeiro ( $\mathrm{N}=695)$. Estudo Pró-Saúde, 1999.

Table2 - Prevalence of insomnia complaints and crude and adjusted odds ratios (OR) and respective $95 \%$ confidence intervals $(95 \% \mathrm{Cl})$ for the association between stressful life events and insomnia complaints among nursing assistants from a university hospital in Rio de Janeiro(N=695). The Pro-Saude Study, 1999.

\begin{tabular}{|c|c|c|c|c|c|c|c|}
\hline \multirow[t]{3}{*}{ EVPE } & \multirow{3}{*}{$\begin{array}{l}\text { Sem queixas } \\
\text { de insônia } \\
\mathrm{N}(\%)\end{array}$} & \multicolumn{6}{|c|}{ Queixas de Insônia } \\
\hline & & \multicolumn{3}{|c|}{ Ocasional } & \multicolumn{3}{|c|}{ Frequente } \\
\hline & & $N(\%)$ & $\begin{array}{l}\text { OR Brutos } \\
\text { (IC 95\%) }\end{array}$ & $\begin{array}{l}\text { OR Ajustados* } \\
\text { (IC 95\%) }\end{array}$ & $\mathrm{N}(\%)$ & $\begin{array}{l}\text { OR Brutos } \\
\text { (IC 95\%) }\end{array}$ & $\begin{array}{l}\text { OR Ajustados* } \\
\text { (IC 95\%) }\end{array}$ \\
\hline $\begin{array}{l}\text { Problemas } \\
\text { sério de } \\
\text { saúde }\end{array}$ & $78(44,1)$ & $51(28,8)$ & $1,28(0,85-1,91)$ & $1,12(0,71-1,75)$ & $48(27,1)$ & $2,68(1,71-4,19)$ & $2,82(1,73-4,58)$ \\
\hline $\begin{array}{l}\text { Internação } \\
\text { em hospital }\end{array}$ & $36(50,7)$ & $18(25,4)$ & $0,91(0,50-1,66)$ & $0,89(0,47-1,68)$ & $17(23,9)$ & $1,63(0,88-3,03)$ & $1,74(0,90-3,35)$ \\
\hline $\begin{array}{l}\text { Falecimento } \\
\text { de parente } \\
\text { próximo }\end{array}$ & $38(41,8)$ & $32(35,2)$ & $1,66(1,00-2,75)$ & $1,59(0,94-2,70)$ & $21(23,1)$ & $1,98(1,11-3,53)$ & $1,61(0,86-3,02)$ \\
\hline $\begin{array}{l}\text { Dificuldade } \\
\text { financeira } \\
\text { grave }\end{array}$ & $185(46,4)$ & $132(33,1)$ & $1,93(1,35-2,74)$ & $1,87(1,27-2,75)$ & $82(20,6)$ & $2,46(1,57-3,86)$ & $2,38(1,46-3,88)$ \\
\hline $\begin{array}{l}\text { Mudança } \\
\text { forçada de } \\
\text { moradia }\end{array}$ & $32(42,7)$ & $24(32,0)$ & $1,46(0,84-2,56)$ & $1,16(0,63-2,15)$ & $19(25,3)$ & $2,13(1,15-3,92)$ & $1,97(1,02-3,79)$ \\
\hline $\begin{array}{l}\text { Rompimento } \\
\text { de relação } \\
\text { amorosa/ } \\
\text { Divórcio }\end{array}$ & $47(35,6)$ & $49(37,1)$ & $2,28(1,46-3,55)$ & $2,30(1,42-3,74)$ & $36(27,3)$ & $3,18(1,93-5,23)$ & $3,32(1,90-5,78)$ \\
\hline $\begin{array}{l}\text { Assalto ou } \\
\text { roubo }\end{array}$ & $51(51,0)$ & $30(30,0)$ & $1,13(0,70-1,85)$ & $1,05(0,62-1,80)$ & $19(19,0)$ & $1,26(0,71-2,24)$ & $1,26(0,68-2,35)$ \\
\hline $\begin{array}{l}\text { Vítima de } \\
\text { agressão } \\
\text { física }\end{array}$ & $15(45,5)$ & $8(24,2)$ & $1,00(0,42-2,41)$ & $0,90(0,38-2,55)$ & $10(30,3)$ & $2,30(1,00-5,27)$ & $2,27(0,92-5,56)$ \\
\hline
\end{tabular}




\section{Discussão}

A prevalência de $45,8 \%$ de queixas de insônia - $16,7 \%$ e $29,1 \%$ para queixas frequentes e ocasionais, respectivamentefoi alta, se comparada a outro estudo com profissionais da enfermagem, como o de Kageyama et al. (2001) ${ }^{29}$, cuja estimativa de prevalência de insônia foi de $29,2 \%$. O fato de a nossa população ser predominantemente feminina $(79,9 \%)$ pode explicar em parte a elevada prevalência de queixas de insônia em nosso estudo. Por outro lado, conforme já foi salientado por outros autores, a utilização de critérios menos rígidos para definir a insônia pode ter contribuído para a superestimação da prevalência. Por exemplo, Morin e cols. (2006) ${ }^{7}$ apontam que a estimativa de que um terço da população adulta apresenta sintomas de insônia (dificuldade para pegar no sono ou manter o sono) se reduz para cerca de $10 \%$, quando são consideradas as consequências diurnas da insônia (fadiga, por exemplo).

Dos oito EVPE estudados, quatro mostraram associação estatisticamente significativa com queixas de insônia: "rompimento de relação amorosa", "problemas graves de saúde", "ter passado por dificuldades financeiras" e "mudança forçada de moradia", mesmo após ajuste para variáveis socioeconômicas, demográficas e trabalho em regime de plantão. Observa-se, portanto, que não é apenas a presença do estresse, mas o tipo de evento vivido, da mesma forma que o observado por Tjekpema (2005) ${ }^{30}$ em amostra representativa da população canadense. Nossos resultados corroboram os de Healey et al. (1981) ${ }^{15}$, segundo os quais as pessoas que referem queixas de insônia relatam maior número de eventos indesejáveis, particularmente relacionados a perdas ou doenças. Pesquisa da National Sleep Foundation, em conjunto com a Gallup Organization $^{6}$, realizada com americanos adultos que sofriam de insônia ocasional mostra que entre os eventos mencionados como causadores de dificuldades para dormir estão o estresse no trabalho (28\% dos indivíduos), o estresse familiar (20\%) e morte de familiares (12\%).

Deve-se ressaltar que os fatores relacionados ao estresse que podem contribuir para o aparecimento da insônia primária geralmente não são levados em consideração quando o paciente procura ajuda médica. Os tratamentos medicamentosos atuam no sintoma (insônia) sem, contudo, atuar na causa do problema, que persistindo poderá tornar a insônia crônica. Em estudo populacional, Kim e cols. $(2000)^{16}$ mencionam a dificuldade em lidar com o estresse como fator associado à maior prevalência de insônia. Neste contexto, cabe mencionar a observação de Morin e cols. (2003) ${ }^{31}$ de que não é o número de eventos estressores per se que aumenta a vulnerabilidade à insônia, mas a percepção da falta de controle sobre esses eventos.

Um aspecto a ser aqui considerado é que a amostra de estudo se diferencia da população geral por ser constituída por funcionários de uma universidade pública, ou seja, uma população de trabalhadores com estabilidade no emprego, representando assim uma parcela da população que possui um patamar socioeconômico mais elevado. Sabemos que as populações desempregadas, com nível socioeconômico mais baixo ou instabilidade no emprego estão ainda mais sujeitas a eventos de vida estressantes e também a uma maior ocorrência de queixas de insônia ${ }^{16}$, de forma que não há como garantir a generalização desses resultados a outros grupos ou populações.

Por se tratar de estudo seccional, os resultados relativos a um dos itens dos EVPEproblemas sérios de saúde - poderiam advir de causalidade reversa, já que as queixas de insônia poderiam levar a problemas sérios de saúde. No entanto, o fato de o período de referência dos EVPE ser os doze meses anteriores ao preenchimento do questionário $\mathrm{e}$ a avaliação de queixas de insônia se referir às duas semanas anteriores, minimiza em parte esta possibilidade, já que provavelmente apenas uma pequena parcela destes eventos ocorreu durante o período avaliado pelas perguntas sobre queixas de insônia. Por outro lado, como não dispomos de 
informação sobre a duração das queixas de insônia, existe a possibilidade de que outra parcela dessas queixas seja um marcador para insônia crônica, ou pelo menos de maior duração, o que levaria a não descartar a hipótese de causalidade reversa.

Os resultados do presente estudo mostram que a história de eventos de vida produtores de estresse nos 12 meses anteriores associa-se a uma maior prevalência de queixas frequentes de insônia entre os auxiliares de enfermagem. A importância de se identificar os principais fatores associados a queixas de insônia entre auxiliares de enfermagem se deve ao fato de essa categoria ter, durante o seu horário de trabalho, a responsabilidade por vidas humanas e em um ambiente onde um acidente de pequeno porte pode pôr em risco a vida das pessoas sob seus cuidados.

\section{Referências}

1. Roth T, Roehrs T, Silva JAC, \& Chase MH. 1999. Public health and insomnia: Consensus statement regarding its status and needs for future actions. Sleep 1999; 22(S3): S417-S20.

2. Walsh J, Ustun TB. Prevalence and health consequences of insomnia. Sleep 1999; 22(S3): 427-36.

3. Rocha FL, Costa MFFL. Epidemiologia e impacto dos distúrbios do sono. J Bras Psiquiatr 2000; 5: 167-80.

4. Léger D, Guilleminault C, Bader G, Lévy E, Paillard M. Medical and socio-professional impact of insomnia. Sleep 2002; 25(6): 621-5.

5. NCSDR (National Center on Sleep Disorders Research). 2003. National sleep disorders research plan. US Department of health and human services national institute of health. Disponível em http://www.nhlbi.nih. gov/health/prof/sleep/res_plan/index.html [Acessado em 24 de outubro de 2004]. National Hearth, Lung, and Blood Institute.

6. Ancoli-Israel SI, Roth T. Characteristics of insomnia in the United States: Result of the 1991 National Sleep Foundation Survey I. Sleep 1999; 22(2): 347-53.

7. Morin CM, Leblanc M, Daley M, Gregoire Jp, Mérette C. Epidemiology of insomnia: prevalence, self-help treatments, consultations, and determinants of helpseeking behaviors. Sleep Medicine 2006; 7: 123-30.

8. Souza JC, Reimão R. Epidemiologia da Insônia. Psicologia em Estudo, Maringá 2004; 9(1):3-7.

9. Krishnam V, Collop NA. Gender differences in sleep disorders. Curr Opin Pulm Med 2006; 12: 383-9.

10. Zhang B, Wing YK. Sex differences in insomnia: a metaanalysis. Sleep 2006; 29(1): 85-93.

11. Ohayon MM, Partinen M. Insomnia and global sleep dissatisfaction in Finland. J Sleep Res 2002; 11: 339-46.
12. Rocha FL, Guerra HL, Costa MFFL. Prevalence of insomnia and associated socio-demographic factors in a Brazilian community: the Bambuí study. Sleep Medicine 2002; 3: 121-6.

13. Giglio SB. Estudo da ocorrência das queixas de insônia, de sonolência excessiva diurna e das relativas às parassonias na população adulta da cidade de São Paulo [tese de doutorado em Farmacologia Não-Publicada]. São Paulo: Curso de Pós-Graduação em Farmacologia de Escola Paulista de Medicina; 1988.

14. Souza JC, Magna LA, Reimão R. Insomnia and hypnotic use in Campo Grande general population, Brazil. Arq Neuropsiquiatr 2002; 60(3-B): 702-7.

15. Healey ES, Kales A, Monroe LJ, Bixler EO, Chamberlin K, Soldatos CT. 1981. Onset of insomnia: role of life-stress events. Psychosomatic Medicine 1981; Oct; 43(5): 439-51.

16. Kim K, Uchiyama M, Okawa M, Liu X, Ogihara R. An epidemiological study of insomnia among the Japanese general population. Sleep 2000; 23(1): 1-7.

17. Kawada T, Yosiaki S, Yasuo K, Suzuki S. Population study on the prevalence of insomnia and insomnia-related factors among Japanese women. Sleep Medicine 2003; 4: 563-7.

18. Drake C, Roehers T, Roth T. Insomnia causes, consequences, and therapeutics: an overview. Depress Anxiety 2003; 18: 163-70.

19. Monti JM. Insônia primária: diagnóstico diferencial e tratamento. Rev Bras Psiquiatr 2000; 22(1): 31-4.

20. Faerstein E, Chor D, Lopes CS, Werneck GL. Estudo PróSaúde: características gerais e aspectos metodológicos. Rev Bras Epidemiol 2005; 8(4): 454-66.

21. Faerstein E, Lopes CS, Valente K, Plá MAS, Ferreira MB. Pré-testes e um questionário multidimensional autopreenchível: A experiência do Estudo Pró-Saúde UERJ. PHYSIS: Rev Saúde Coletiva 1999; 9(2): 117-30. 
22. APA (American Psychiatric Association). Diagnostic and Statistical Manual of Mental Disorders: fourth edition (DSM-IV). Washington: American Psychiatric Association; 1994.

23. AASM (American Academy of Sleep Medicine). International Classification of Sleep Disorders, Revised: Diagnostic and Coding Manual. Chicago, Illinois: American Academy of Sleep Medicine; 2001.

24. OMS (Organização mundial da Saúde). Classificação dos Transtornos Mentais e de Comportamento da CID-X: referência rápida. (Vol 1). Porto Alegre: Artes Médicas; 1997.

25. Ohayon MM. Epidemiology of insomnia: what we know and what we still need to learn. Sleep Medicine 2002; 6(2):97-111.

26. Lopes CS, Faerstein E. Confiabilidade do relato de eventos de vida estressantes em um questionário autopreenchido: Estudo Pró-Saúde. Rev Bras Psiquiatr 2001; 23(3): 126-33.
27. Lopes CS, Faerstein E, Chor D. Eventos de vida produtores de estresse e transtornos mentais comuns: resultados do Estudo Pró-Saúde. Cad Saude Publica 2003; 19 (x): 109-18.

28. Hosmer DW, Lemeshow S. Applied Logistic Regression. New York: John Wiley \& Sons; 1989.

29. Kageyama T, Nishikido N, Kobayashi T, Oga J, Kawashima M. Cross-sectional survey on risk factors for insomnia in Japanese female hospital nurses working rapidly rotating shift systems. J Hum Ergol 2001; 30: 14954.

30. Tjepkema M. Health Statistics Division, Statistics Canada, Toronto Regional Office, Ontario. Health Rep 2005; 17(1): 9-25.

31. Morin CM, Rodrigue S, Ivers H. Role of stress, arousal, and coping skills in primary insomnia. Psychosom Med 2003; 65: 259-67.

Recebido em: 29/04/09

Aprovado em: 08/07/09 